\title{
Characterization of extreme precipitation within atmospheric river events over California
}

\author{
S. Jeon ${ }^{1}$, Prabhat ${ }^{2}$, S. Byna ${ }^{2}$, J. Gu ${ }^{2}$, W. D. Collins ${ }^{1,3}$, and M. F. Wehner ${ }^{2}$ \\ ${ }^{1}$ Earth Sciences Division, Lawrence Berkeley National Laboratory, Berkeley, California, USA \\ ${ }^{2}$ Computational Research Division, Lawrence Berkeley National Laboratory, Berkeley, California, USA \\ ${ }^{3}$ University of California, Berkeley, Berkeley, California, USA
}

Correspondence to: S. Jeon (soyoungj82@gmail.com)

Received: 2 March 2015 - Revised: 21 October 2015 - Accepted: 24 October 2015 - Published: 17 November 2015

\begin{abstract}
Atmospheric rivers (ARs) are large, spatially coherent weather systems with high concentrations of elevated water vapor. These systems often cause severe downpours and flooding over the western coastal United States - and with the availability of more atmospheric moisture in the future under global warming we expect ARs to play an important role as potential causes of extreme precipitation changes. Therefore, we aim to investigate changes in extreme precipitation properties correlated with AR events in a warmer climate, which are large-scale meteorological patterns affecting the weather and climate of California.

We have recently developed the TECA (Toolkit for Extreme Climate Analysis) software for automatically identifying and tracking features in climate data sets. Specifically, we can now identify ARs that make landfall on the western coast of North America. Based on this detection procedure, we can investigate the impact of ARs by exploring the spatial extent of AR precipitation using climate model (CMIP5) simulations and characterize spatial patterns of dependence for future projections between AR precipitation extremes under climate change within the statistical framework. Our results show that AR events in the future RCP (Representative Concentration Pathway)8.5 scenario (2076-2100) tend to produce heavier rainfall with higher frequency and longer days than events from the historical run (1981-2005). We also find that the dependence between extreme precipitation events has a shorter spatial range, within localized areas in California, under the high future emissions scenario than under the historical run.
\end{abstract}

\section{Introduction}

Atmospheric rivers (ARs) are narrow bands of elevated tropospheric water vapor that are thousands of kilometers long and hundreds of kilometers wide. ARs have generated recent interest because they have been proven capable of producing extreme precipitation and flood damage over the western coastal United States. Ralph et al. (2005) define ARs in California as containing high amounts of water vapor and strong winds that force this vapor to higher altitudes in coastal or mountainous terrain resulting in heavy precipitation. Atmospheric rivers often result in extreme winds and precipitation. For instance, Ralph et al. (2006) document a specific AR event that produced more than $10 \mathrm{in}$. of rainfall in 2 days and caused extensive flooding near the Russian River in northern California. One particularly damaging class of ARs, popu- larly called the "pineapple express" has been implicated in several recent major storms along the west coast of the US (Dettinger, 2004; Neiman et al., 2008; Dettinger, 2011).

In order to collect statistics on AR events, we implemented a pattern detection scheme in TECA (Toolkit for Extreme Climate Analysis) (Prabhat et al., 2012; Byna et al., 2011). Using high performance computing platforms, TECA enables efficient extraction of climate patterns in large data archives such as those generated by the Coupled Model Intercomparison Project Phase 5 (CMIP5). In this paper, we implemented the AR detection procedure described by existing literature (Ralph et al., 2004; Neiman et al., 2008; Wick et al., 2013) and applied it to historical and future simulations in CMIP5. The detection results presented in Wick et al. (2013) are fairly convincing, and we did not feel the necessity to innovate further on the detection procedure. Instead, we fo- 
cused on the large-scale application of the existing technique in TECA and on the characterization of the extreme precipitation resulting from the simulated ARs.

There are relatively few studies concerning the spatial variability of precipitation caused by the landfall of ARs and the impact of ARs on extreme precipitation under a changing climate. Leung and Qian (2009) investigated the influence of ARs with respect to the spatial distribution of precipitation for two events, Presidents' Day (US) in 1986 and New Year's Day in 1997, when AR precipitation anomalies were observed. Rutz et al. (2014) analyzed the spatial distribution of AR duration, AR frequency, and fraction of precipitation from land-falling ARs along the west coast of the United States. Precipitation extremes in the CMIP5 ensemble increase for some regions with global warming, and the return periods of extreme precipitation events become shorter under the higher radiative forcing scenarios by the end of the $21 \mathrm{st}$ century, but the pattern of changes in extreme precipitation properties varies with different regions and different models (Sillmann et al., 2013b; Kharin et al., 2013). One might expect that the frequency and intensity of ARs would change with the increasing atmospheric moisture caused by global warming. In this study, our extreme precipitation metric is the AR version of $R \times 1$ day. This CMIP5 ensemble average of this field over California increases, particularly in the northern and central portion of the state under CMIP5. North American decreases in the indices by Sillmann et al. (2013b) are more prominent in the dry subtropics and are especially apparent in Mexico. Along these lines, Dettinger (2011) investigated seven global circulation models (GCMs) for historical and future climate simulations and showed that AR storms in California become more frequent and more severe under a warming (A2 emissions) scenario in the future projections. Lavers et al. (2013) recently analyzed the projected AR changes in five CMIP5 GCMs for the RCP (Representative Concentration Pathway)8.5 (high emissions) scenario and showed that future Atlantic ARs exhibit increased water vapor with a higher risk of heavy rainfall in Britain.

Climate extremes can be analyzed via a specific statistical theory, the so-called extreme value theory, to quantify the distribution of extreme values (e.g., annual maximum, annual minimum, or excesses over high thresholds) and the probability of the rare events (e.g., Coles, 2001). Generalized extreme value (GEV) and peaks-over-threshold (POT) techniques have been successfully applied to extreme meteorological events to analyze the return values of extreme temperature or precipitation events (Kharin et al., 2007; Coelho et al., 2008; Kharin et al., 2013; Wehner, 2013). Statistical methods (in particular point process approaches) based on extreme value analysis have also been applied to the characterization of trends in hot spells and heat waves (Furrer et al., 2010).

In spatial extreme value analysis, multivariate extreme value theory analyzes the dependence of extremes at multiple locations. Max-stable processes (in the framework of stochastic processes) have been applied to model joint distribution of spatial extremes and their dependence (de Haan, 1984, Smith, unpublished data). Cooley et al. (2006) developed an estimator to measure the degree of spatial dependence between extremes at two locations. In another study by Bernard et al. (2013), heavy rainfall in France was spatially clustered based on the measurement of spatial dependence. Weller et al. (2012) investigated the tail dependence in daily precipitation between reanalysis output and observations of pineapple express events using bivariate extreme value theory.

The novel aspects of our study follow from the statistical analysis of extreme precipitation associated with ARs using a concept of a spatial dependence structure. Here, we quantify spatial extremal dependence to analyze extreme precipitation events caused by ARs associated with large-scale coherent weather systems, focusing on the California region. This study provides detailed characterizations of changes in AR properties and the spatial dependence of extreme rainfall under AR conditions in projections of future climate change. Our principal motivation behind this study is to better understand the characteristics of extreme precipitation in ARs in a warming scenario. As the physical mechanisms causing different types of storms differ, it can be expected that their statistical descriptions will differ as well. In Sect. 2, we introduce the framework of TECA developed at the Lawrence Berkeley National Laboratory as an AR identifier, describe our methodology for spatial extreme value analysis, and introduce our metric of spatial dependence. We focus on the characterization of spatial properties in extreme precipitation from ARs identified in ensembles of climate simulations from CMIP5. Section 3 discusses overall trends in AR events and extreme precipitation, as well as the results from the analysis of spatial dependence between precipitation extremes during AR events. Our conclusions and further discussion are presented in Sect. 4.

\section{Methodologies}

\subsection{Characteristics of atmospheric rivers}

ARs play a prominent role in the climatology of inland precipitation over the western United States. Ralph et al. (2005) investigated the structure of AR events over the eastern Pacific Ocean using dropsonde observations. ARs are typically 2000 or more kilometers long and a few hundred kilometers wide, and the integrated water vapor (IWV) in the atmosphere during AR events is greater than $20 \mathrm{~mm}$, producing heavy rainfall; for example $>250 \mathrm{~mm}$ of rain in $60 \mathrm{~h}$ for a storm in February 2004 in northern California (Ralph et al., 2006; Neiman et al., 2008). Warner et al. (2012) used National Climate Data Center (NCDC) daily precipitation observations for 1950-2009 to investigate the top 50 storm events in 2-day precipitation at six coastal stations (a total of 207 events in the top 50 from 60 years and multiple sta- 


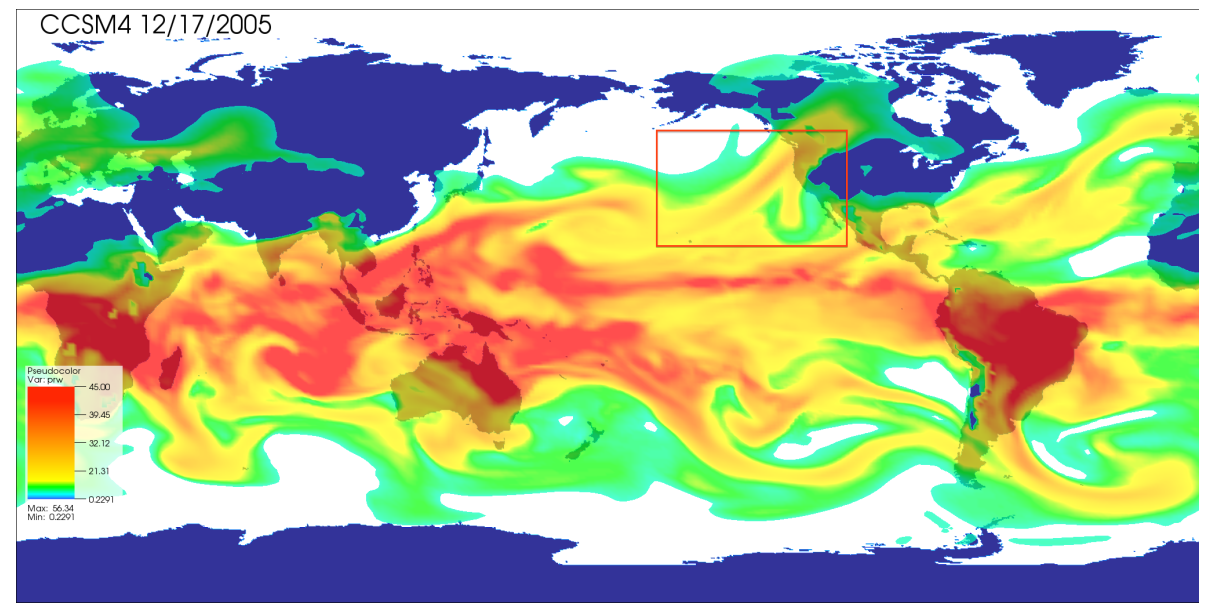

Figure 1. Total daily precipitable water (prw) field $\left(\right.$ in $\mathrm{kg} \mathrm{m}^{-2}$ ) from a sample of the CCSM4 model output. It shows a typical AR that originated at the tropical region near Hawaii as it reaches the western coast of the US and is identified by the TECA detection procedure.

tions) along the Pacific northwest coast. From NCEP-NCAR reanalysis data in Warner et al. (2012), most events are associated with ARs and the extreme precipitation events typically occur in November, December, and January, producing heavy rainfall for the periods from 16 to $86 \mathrm{~h}$. Payne and Magnusdottir (2014) characterized landfalling ARs along the west coast of North America for the extended winter (November-March) during 1979-2011 using ModernEra Retrospective Analysis for Research and Applications (MERRA) reanalysis data. The largest number of landfalling AR dates during the season occur in November (6.1 days, averaged for total dates of the month), December (5.8 days), and January (5.2 days). The most intense landfalling dates occur in November $\left(302.3 \mathrm{~kg} \mathrm{~m}^{-1} \mathrm{~s}^{-1}\right.$, peak daily moisture flux), with less intense AR dates later in the extended winter over the western coastline of North America. Rutz et al. (2014) indicates that AR-related precipitation is strongly influenced by three factors: climatological characteristics of ARs along the US west coast, water vapor depletion over high mountain barriers, and increase in surface elevation and decrease in pressure. Lavers et al. (2012) used atmospheric reanalysis products for the detection of ARs, and linked the steep slopes of basins and basin orientation to the high precipitation delivered by the ARs. Lundquist et al. (2010) discussed the formation of sierra barrier jets (SBJ) when the atmospheric flow is steered to be roughly parallel to the mountain range.

\subsection{Detection of atmospheric rivers}

Our AR detection code (described in detail in Byna et al., 2011) utilizes the following scheme for detecting ARs. We first compute a 2-D total-column-integrated precipitable water (prw) field by performing a vertical integral on the specific humidity (hus) field. The vertical integral of hus was performed over all of the prescribed standard output pressure layers as daily average values of prw were not part of the CMIP5 protocols. The integration was done for the full vertical extent of the modeled atmospheres, but any levels above the tropopause contribute very little to prw. The integration is performed on daily data, and the detections are reported on a daily basis as well. We tracked AR only over the northern Pacific Ocean region in order to focus our analysis on ARs that intersect with the California coastline. Following the definition of the physical features of an AR (Ralph et al., 2004; Neiman et al., 2008), we conduct a thresholding operation for identifying all grid points with prw $>2 \mathrm{~cm}$, the threshold taken from existing literature. We then use a connected component labeling algorithm to find all of the connected regions of grid points satisfying this criterion for elevated precipitable water. Our implementation of connected component labeling is based on a two-pass algorithm described in Wu et al. (2009) and Byna et al. (2011). Finally, we apply geometric constraints to the resulting merged polygons (connected components), which represent a set of candidate atmospheric rivers. These geometric constraints trace prw from a departure point from the tropics (at the west side of the feature) to the intersection with the west coast of North America. Hence, the ARs considered in this study are a subset of all possible ARs across the globe. For all the polygons satisfying these origination and termination conditions, we first construct a medial axis, then compute the length parallel and width perpendicular to this axis, and finally check whether the length of the AR $>2000 \mathrm{~km}$ and if the width of the $\mathrm{AR}<1000 \mathrm{~km}$. If a polygon satisfies all of these geometric constraints, we identify it as an atmospheric river and note the date of the occurrence. In Fig. 1, we show a sample AR event detected in the CCSM4 model data (highlighted in the red box) using the AR detection code of TECA. The AR is identified on the simulated date of 17 December 2005, where the landfall points of the $\mathrm{AR}$ are $\left(29.686^{\circ} \mathrm{N}, 125.000^{\circ} \mathrm{W}\right)$ and $\left(44.764^{\circ} \mathrm{N}, 126.250^{\circ} \mathrm{W}\right)$ in the latitude and longitude 
Table 1. The CMIP5 models, modeling groups, and ensemble member(s) used for historical (1981-2005) and RCP8.5 runs (2076-2100) in the study.

\begin{tabular}{clll}
\hline Number & Model & Modeling group & Ensemble runs \\
\hline 1 & CanESM2 & Canadian Centre for Climate Modelling and Analysis & 4 \\
2 & CCSM4 & National Center for Atmospheric Research & 1 \\
3 & GFDL-ESM2M & NOAA Geophysical Fluid Dynamics Laboratory & 1 \\
4 & IPSL-CM5A-LR & Institut Pierre-Simon Laplace & 4 \\
5 & IPSL-CM5A-MR & Institut Pierre-Simon Laplace & 1 \\
6 & MIROC5 & AORI (Atmosphere and Ocean Research Institute),, & 2 \\
& & NIES (National Institute for Environmental Studies), & 1 \\
7 & MIROC-ESM-CHEM & JAMSTEC (Japan Agency for Marine-Earth Science and Technology), Japan & AORI (Atmosphere and Ocean Research Institute), \\
& & NIES (National Institute for Environmental Studies), & 3 \\
8 & MPI-ESM-LR & JAMSTEC (Japan Agency for Marine-Earth Science and Technology), Japan & 1 \\
9 & MRI-CGCM3 & Max Planck Institute for Meteorology & 1 \\
\hline Totals & NorESM1-M & Neteorological Research Institute & 19 \\
\hline
\end{tabular}

coordinates, the width of the AR is $657.713 \mathrm{~km}$, and the length of the AR is $7529.872 \mathrm{~km}$. All further processing considers analysis of precipitation values in California for those specific dates in which ARs are detected.

\subsection{Data description}

In this analysis, we consider two variables from CMIP5 models for the historical and RCP8.5 (the most aggressive warming scenario considered in the CMIP5) experiments: (1) daily hus (the mass fraction of water vapor in moist air, in $\mathrm{kg} \mathrm{kg}^{-1}$ ) to obtain total prw by integrating hus in the vertical dimension over all available vertical model levels for AR detection; and (2) daily precipitation (pr, precipitation flux, in $\mathrm{kg} \mathrm{m}^{-2} \mathrm{~s}^{-1}$ ) to characterize spatial properties of heavy rainfall under AR events. These two variables were extracted from the Earth System Grid Federation (ESGF) archive for 10 CMIP5 multi-models (listed in Table 1) with all available ensemble members, which were used to account for the uncertainty of each model experiment. For each model, pr was regridded to a common grid of resolution $1.25^{\circ} \times 0.94^{\circ}$ to aid in comparison. The performance of CMIP5 models in simulating extreme precipitation from all types of storms is evaluated in more detail (Sillmann et al., 2013a; Kharin et al., 2013). Langford et al. (2014) examined mean precipitation variability in CMIP5 historical simulations for the California region. The CMIP5 models simulate higher mean precipitation on average than reanalysis data sets, and some models (IPSL-CM5B-LR, MRI-CGCM3, and GISS) show high temporal variability on average in the winter months, when a peak in precipitation typically occurs in the California region. Therefore, discrepancies between model and observations or their proxy products would need to be assessed carefully before usage by decision makers.
Our goal in this study is to explore how the frequency and duration of ARs change in future, warmer climates together with the resulting changes in the spatial properties of extreme precipitation events. Changes in the statistics of AR events and extreme precipitations are explored by comparing two 25-year time periods spanning 1981-2005 and 2076-2100. Here, we use the calendar year from 1 January to 31 December and treat an AR event that bridges the new year as the event in the year in which it starts. We focus on the occurrence of ARs in California and note that most AR events in California occur in winter and spring. For selected grid points in California, we take the maximum value from the time series of daily precipitation only for the dates in which ARs are detected within a given year and define this as the annual maximum AR precipitation. In order to compare ARrelated extreme precipitation against overall extreme precipitation driven by all sources of events, we also compute the annual maximum precipitation by taking the maximum value from daily precipitation during the entire year at each grid point.

\subsection{Spatial extreme value analysis}

We use spatial extreme value analysis to quantify the relationships between extreme events occurring at different locations on different days in the same year. Dependence between extremes is affected by the geographic location. Spatial covariance structure is useful for modeling the dependence between spatially distributed variables where the correlations are defined as a function of distance. Under a spatial analysis framework, one can characterize dependence between extremes at two locations by making the modeling assumption regarding the covariance structure (the details of the covariance structure are be described later on). Spatial dependence 
structure in this study describes whether extremes at different sites, which may occur from distinct ARs during the whole time period, show more or less similarity when the sites are physically close to or far apart from each other.

One appropriate statistical methodology for modeling spatial extremes is the max-stable process approach. Max-stable processes have been used to stochastically model the joint distribution of extreme values at multiple sites (de Haan, 1984; Schlather, 2002; Kabluchko et al., 2009, Smith, unpublished data). The process works as follows: let $X\left(s_{i}\right)$ denote a spatial process in which climate variables such as daily precipitation are observed at locations $\boldsymbol{s}_{i}(i=1, \ldots, n)$ on a spatial domain. Then, after suitable normalization of $X(s)$, the maximum of the normalized process, for example annual or seasonal maximum precipitation, converges in probability distribution to a process $Y(\boldsymbol{s})$ :

$$
\max _{i=1, \ldots, n} \frac{X\left(s_{i}\right)-b_{n}(s)}{a_{n}(s)} \rightarrow Y(s),
$$

and the limit process $Y(s)$ is called a max-stable process. In the max-stable modeling approach, spatial dependence can be characterized by a covariance structure between spatial processes at different locations, which is specified by a variogram. The variogram is a function of distance between (typically two) spatial locations and is commonly used for modeling the spatial variance and correlation in geostatistics. Here, we assume an isotropy of spatial processes and the variogram function depends only on the distance of locations. In the modeling procedure, the covariance structure (referred as dependence structure) is modeled by fitting max-stable processes with a parametric form of the variogram function (i.e., a function of parameters), and we estimate the parameters of the variogram to maximize the likelihood of a max-stable model for extreme events.

For example, Kabluchko et al. (2009) show how a class of max-stable processes known as Brown-Resnick processes can be used to model extreme phenomena at multiple locations by the Brownian stochastic process. For the BrownResnick process with the dependence structure modeled by a variogram $\gamma$, the bivariate probability distribution is formulated as follows:

$$
\begin{gathered}
P\left(Y\left(\boldsymbol{s}_{1}\right) \leq y_{1}, Y\left(\boldsymbol{s}_{2}\right) \leq y_{2}\right) \\
\quad=\exp \left\{-\frac{1}{y_{1}} \Phi\left(\frac{\sqrt{\gamma(h)}}{2}+\frac{1}{\sqrt{\gamma(h)}} \log \frac{y_{2}}{y_{1}}\right)\right. \\
\left.\quad-\frac{1}{y_{2}} \Phi\left(\frac{\sqrt{\gamma(h)}}{2}+\frac{1}{\sqrt{\gamma(h)}} \log \frac{y_{1}}{y_{2}}\right)\right\},
\end{gathered}
$$

where $\Phi$ is the standard normal distribution function, and $h$ is the Euclidean distance between locations $\boldsymbol{s}_{1}$ and $\boldsymbol{s}_{2}$. When an exponential variogram, i.e., $\gamma(h)=\theta_{0}\left(1-e^{-h / \theta_{1}}\right)$, is used in the process of fitting the max-stable model, we parameterize the variogram function with two parameters: (1) $\theta_{0}$, the sill parameter, describing the variance in the random field; and (2) $\theta_{1}$, the range parameter, representing the range of dependence.

An extremal coefficient provides numerical values in a specific interval for the measurement of dependence between extremes, while the estimation of variogram functions provide information on the covariance structure of spatial processes for extremes. The extremal coefficient quantifies the degree of spatial dependence for extremes at different locations and is based on the multivariate extreme value theory (Schlather and Tawn, 2003). The pairwise extremal coefficient, widely used for expressing the dependence between a pair of extremes at two locations, takes values between 1 (complete dependence) and 2 (complete independence). Smith (unpublished data) proposed a naive estimator of the pairwise extremal coefficient. Cooley et al. (2006) and Naveau et al. (2009) investigated a non-parametric approach in characterizing the pairwise dependence among maxima. In this study, we apply the approach by Cooley et al. (2006) to assess pairwise dependence.

To summarize our methodology, we first identify those AR events, via the TECA detection procedure, that make landfall in California in the CMIP5 simulations. We then characterize the changes in total AR days and AR frequency by comparing the intensity of extreme precipitation in presentday and future runs conditioned on the occurrence of ARs. We also characterize the spatial patterns of dependence for future projections under climate change within the framework of extreme value theory. For the application of spatial tail dependence, we fit the Brown-Resnick max-stable processes with a power law variogram, $\gamma(h)=\theta_{1} h^{\theta_{2}}$, and quantify the dependence structure by estimating the parameters, $\theta_{1}$ (range parameter) and $\theta_{2}$ (smoothing parameter, representing the smoothness of the variogram function). We also estimate the extremal coefficients using the madogram estimator developed by Cooley et al. (2006) to determine the pairwise dependence of extreme AR precipitation between a focal point and other grid points. The analysis of spatial dependence provides useful information of how extreme values measured at different locations will be correlated spatially. We can also find changes in spatial dependence between extremes induced by AR events in a changing climate, implying changes in spatial coherence of dependence structure between extremes within a warmer climate.

\section{Results}

\subsection{Overall trend in atmospheric river events}

We examine changes in the overall behavior of ARs using CMIP5 multi-model ensemble simulations in a changing climate under the RCP8.5 emissions scenario. When an AR satisfying the conditions - length and width of merged polygons, and prw exceeding a certain threshold - is detected and it overlaps any portion of the California region, we treat it as an individual AR event. If the detected event lasts more than 


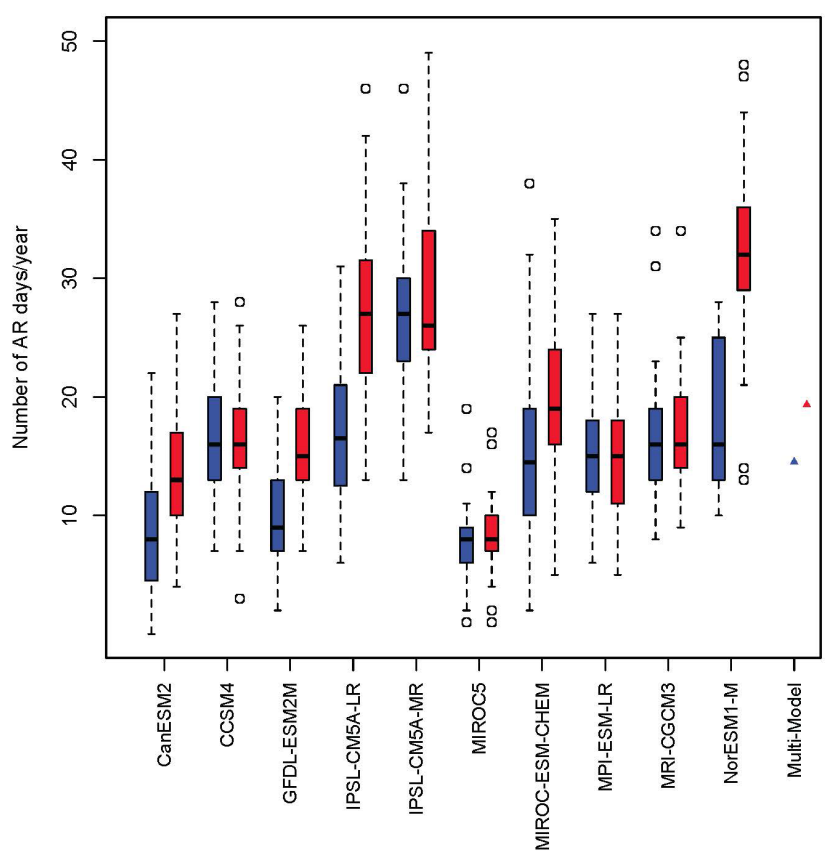

Figure 2. Boxplot summaries of annual values of atmospheric river days (AR days, unit: days $\mathrm{yr}^{-1}$ ) for the California region from each CMIP5 model during the 25 years, spanning 1981-2005 (blue boxplot) and 2076-2100 under RCP8.5 (red boxplot). Blue and red triangles represent the ensemble mean of AR days for the historical and $\mathrm{RCP} 8.5$ runs, respectively.

1 day, it is counted as a single AR event. AR days (i.e., total days of ARs in a year) are counted for two 25-year time periods. The boxplots of AR days for each model, including multi-model ensemble means of AR days, are shown for historical (blue) and RCP8.5 (red) runs in Fig. 2. Changes in the median of the boxplots for each model represent the differences in the median in a warmer climate for the model. Only IPSL-CM5A-MR shows a decrease $\left(2\right.$ days $\left.\mathrm{yr}^{-1}\right)$ in AR days while NorESM1-M shows the largest increase (17 days $\mathrm{yr}^{-1}$ ) of AR days in a warmer scenario. Under the RCP8.5 scenario in years 2076-2100, the ensemble mean of AR days shows substantial increases - on average of $33 \%$ - compared to historical runs in years 1981-2005.

Figure 3 shows boxplots of the number of AR events in historical (blue boxplot) and RCP8.5 (red boxplot) runs for each model. AR frequencies (i.e., total AR events in a year) also increase for all models within the RCP 8.5 scenario by the end of the 21st century, as indicated by median values of boxplots. The NorESM1-M simulation projects the largest change in the number of ARs with 19 events $\mathrm{yr}^{-1}$ for the period 2076-2100 compared to 11 events $\mathrm{yr}^{-1}$ for the period 1981-2005, representing a $73 \%$ increase in AR frequency. Most models show 2-6 more AR events per year under the RCP8.5 scenario than in the historical run.

Yearly statistics of ARs show 12-13 events between 2002 and 2010 from observations by AMSR-E (Advanced

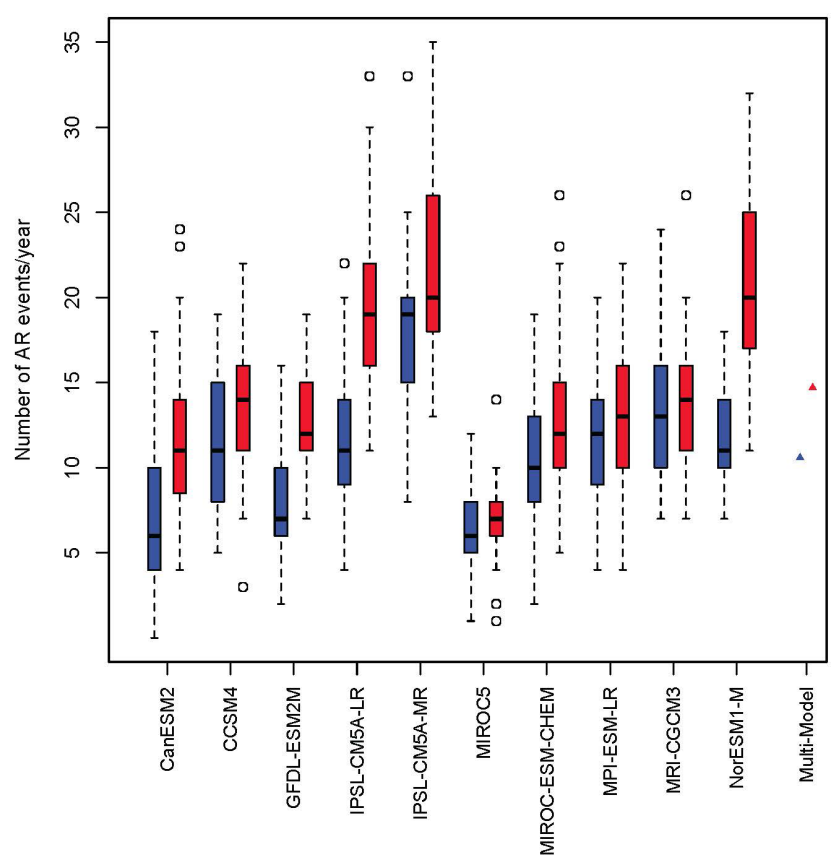

Figure 3. Boxplot summaries of annual numbers of atmospheric river events (AR frequency, unit: events $\mathrm{yr}^{-1}$ ) for the California region from each CMIP5 model during the 25 years, spanning 19812005 (blue boxplot) and 2076-2100 under RCP8.5 (red boxplot). Blue and red triangles represent the ensemble mean of AR counts for the historical and RCP8.5 runs, respectively.

Microwave Scanning Radiometer) satellite data (Fig. 6 in Byna et al., 2011). The ensemble mean of AR counts $\left(10.6\right.$ events $\mathrm{yr}^{-1}$ ) for historical run matches up well with the statistics from the observational data, and the multi-model ensembles show more frequent AR events (14.7 events $\mathrm{yr}^{-1}$, average of $39 \%$ increase) in the future under the RCP 8.5 scenario. In any case, to evaluate model performance in CMIP5 with more accurate detection of ARs, more comparisons of climate simulations and observational data sets need to be performed for consistent time periods. As shown in Figs. 2 and 3, AR events are consistently longer and more frequent under the RCP8.5 scenario (2076-2100) than in the historical run (1981-2005). Lavers et al. (2013) also showed that AR frequency in the northern Atlantic has approximately doubled under the RCP8.5 scenario for the period 2074-2099.

\subsection{Characterization of spatial properties in extreme AR precipitation}

We turn now to the characterization of extreme precipitation during ARs making landfall in California. Figure 4 shows boxplots of the annual maximum AR precipitation (in $\mathrm{mm} \mathrm{day}^{-1}$ ) for each of CMIP5's historical and future RCP8.5 runs. The AR extreme precipitation increases as temperature increases for most of the models. The ensemble mean of the annual maximum AR precipitation changes from 45.1 


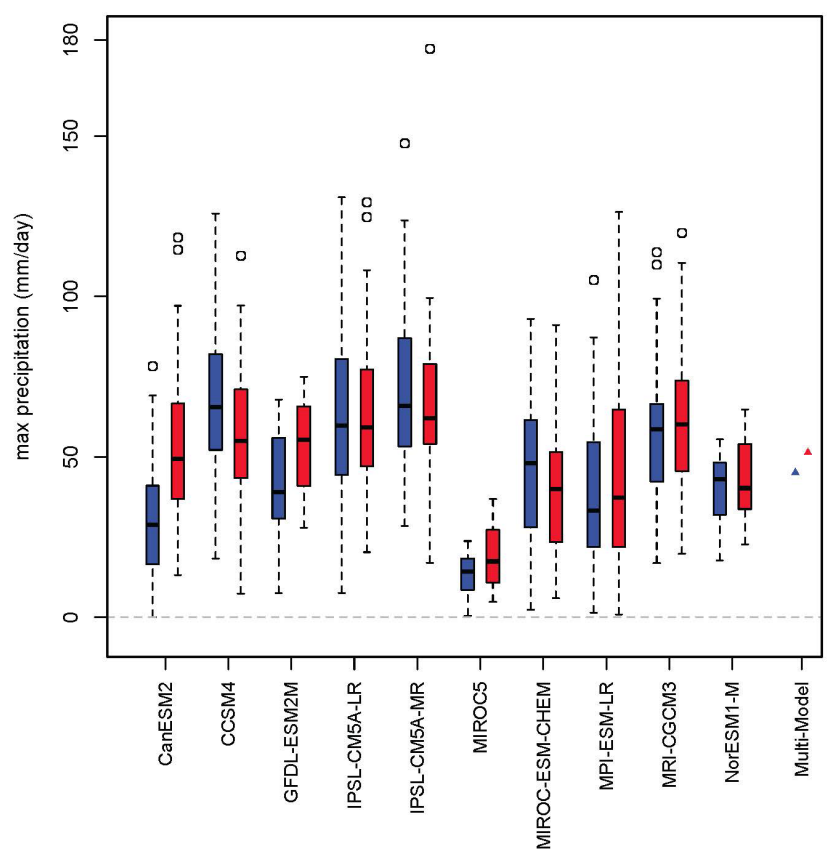

Figure 4. Boxplot summaries of annual maximum AR precipitation (unit: $\mathrm{mm} \mathrm{day}^{-1}$ ) in California from each CMIP5 model, during the 25 years spanning 1981-2005 (blue boxplot) and 2076-2100 under RCP8.5 (red boxplot). Blue and red triangles represent the ensemble mean of AR precipitation extremes for the historical and RCP8.5 runs, respectively.

(blue triangle) to $51.3 \mathrm{~mm} \mathrm{day}^{-1}$ (red triangle), showing a $14 \%$ increase on average. However, the individual models exhibit both increases and decreases. Five models (CCSM4, IPSL-CM5A-LR, IPSL-CM5A-MR, MIROC-ESM-CHEM, and NorESM1-M) show decreases while the other five models (CanESM2, GFDL-ESM2M, MIROC5, MPI-ESM-LR, and MRI-CGCM3) show increases in median values of this AR-related precipitation extreme.

Now we investigate changes in the spatial properties of extreme precipitation associated with ARs in a warmer climate. To find spatial variability between AR extreme precipitations, we summarize the ensemble means of maximum precipitation amounts within the AR events at each grid point. Nine grid points on the common grid are selected among all 34 grid points in California (Fig. 5a) - to represent the spatial pattern of the extreme precipitation throughout southern California (stations 2, 9, and 11), central California near sierras (stations 16 and 18) and northern California (stations 23, 25, 29, and 31). In Fig. 5b, we show boxplots of overall maximum precipitation (i.e., annual maximum precipitation) versus maximum precipitation associated with AR events during 25-year time periods at nine grid points in California.

Increases in maximum precipitation during AR events are consistent with a general pattern toward a warmer climate in the region, but the amount of increases vary spatially - showing relatively larger changes in the extreme rainfall amounts
Table 2. Multi-model ensemble means of range and smoothing parameter estimates, standard deviations, and $95 \%$ confidence intervals for parameters from modeling of Brown-Resnick processes. Range parameter estimates decrease under the future RCP8.5 scenario.

\begin{tabular}{lccc}
\hline Historical period & Mean & $\begin{array}{c}\text { Standard } \\
\text { deviation }\end{array}$ & $\begin{array}{c}95 \% \text { confidence } \\
\text { interval }\end{array}$ \\
\hline Range, $\theta_{1}$ & 4.56 & $(1.39)$ & $(3.94,5.18)$ \\
Smoothing, $\theta_{2}$ & 1.47 & $(0.23)$ & $(1.36,1.57)$ \\
\hline RCP8.5 & & & \\
& Mean & Standard & $95 \%$ confidence \\
& & deviation & interval \\
\hline Range, $\theta_{1}$ & 3.72 & $(1.19)$ & $(3.18,4.25)$ \\
Smoothing, $\theta_{2}$ & 1.59 & $(0.27)$ & $(1.47,1.71)$ \\
\hline
\end{tabular}

for northern California compared to those for southern California. Figure 5b also illustrates the differences between extreme precipitation amounts within AR and non-AR events by grid point. We find that the median values for maximum AR precipitation are always lower than those for annual maximum precipitation in both the historical and future RCP8.5 runs. This is an indication that the AR storms identified by the TECA algorithm do not produce the most extreme precipitation events in the models. It is likely that extratropical cyclones, with cyclogenesis near the Aleutian Islands are responsible for the highest annual daily precipitation totals despite the fact that they are generally colder storms than are ARs of tropical origins. This result may not hold for the averages of all storms in these two classes because of this temperature difference. However, for extreme precipitation events, dynamical considerations can be as important as the thermodynamical. A more detailed analysis of the differences between storm types is outside the scope of this paper (Warner et al., 2012; Grotjahn and Faure, 2008).

From fitting the Brown-Resnick max-stable process to maximum $\mathrm{AR}$ precipitation, the range $\left(\theta_{1}\right)$ and smoothing $\left(\theta_{2}\right)$ parameters in the power law variogram are estimated from each CMIP5 model for modeling of the process, and the ensemble means of those estimates, standard deviations, and $95 \%$ confidence intervals for the parameters are shown in Table 2. The range parameter estimates decrease in the RCP8.5 scenario relative to the present-day simulation, representing less spatially correlated AR precipitation extremes in the future. In other words, the distances over which AR extreme precipitation amounts are correlated decreased, suggesting that correlation between intense AR precipitation patterns are reduced in size. However, the changes in range parameter estimation are not statistically significant under a two-sided permutation test at a $5 \%$ significance level $(p$ value $=0.051)$.

In Fig. 6, we illustrate changes in the spatial extent of dependence between AR precipitation extremes from multi- 
(a)

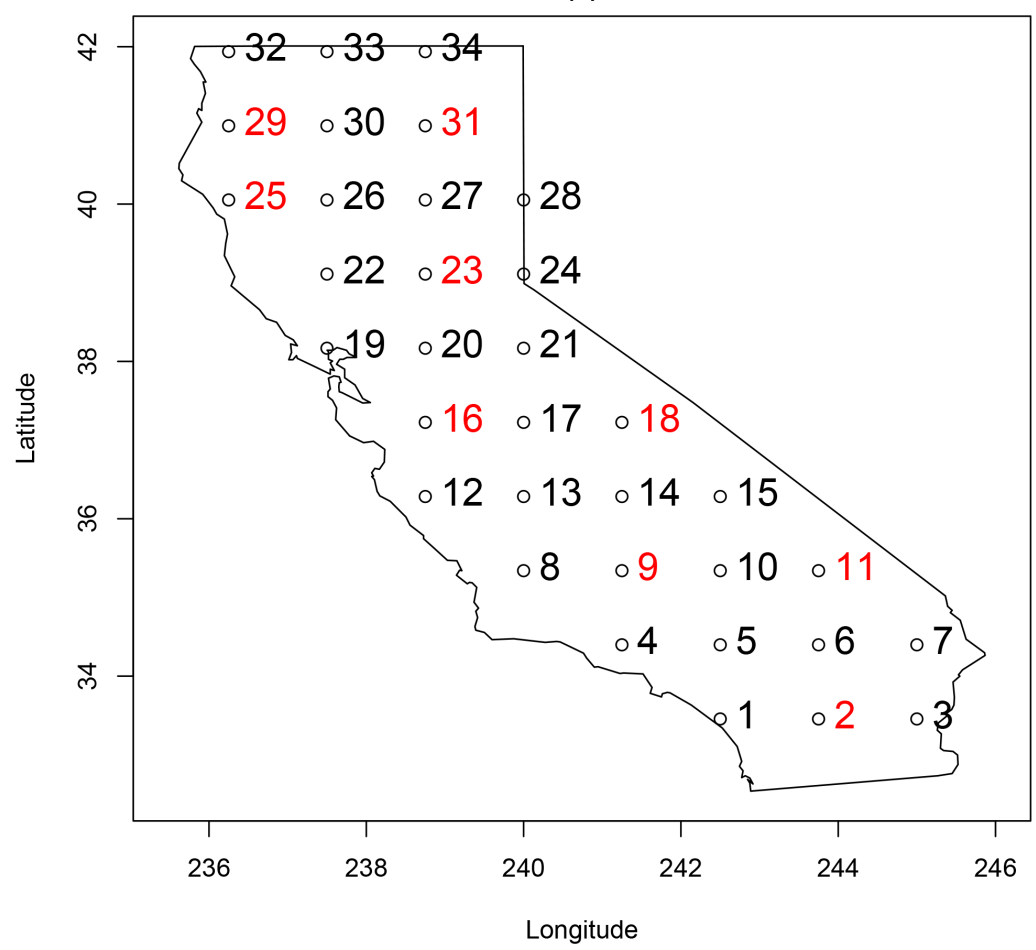

(b)
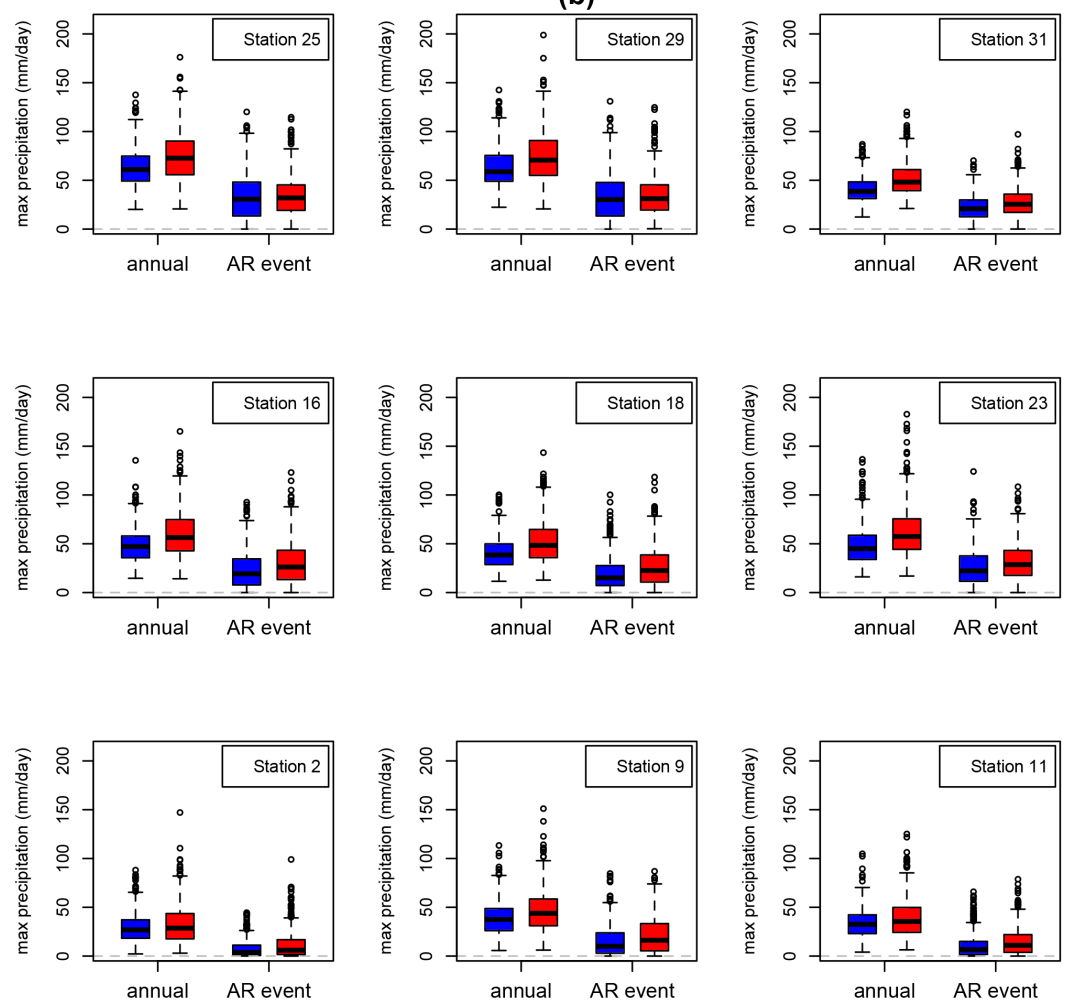

Figure 5. (a) Grid points of CMIP5 models in California, USA, and (b) boxplot summaries of annual maximum precipitation (left pair of box and whiskers at each indicated grid point) and maximum precipitation within AR events (right pair of box and whiskers at each indicated grid point) during 25-year time periods at nine grid points in California. Blue and red boxplots represent the historical and RCP8.5 runs, respectively, and the station numbers are used to identify grid points in red (a). 
(a) Historical run
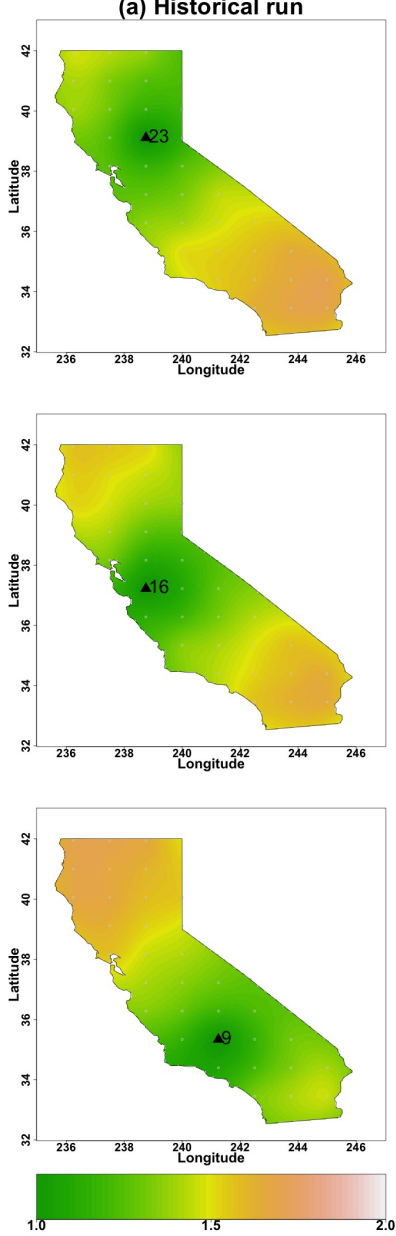

(b) RCP8.5 run
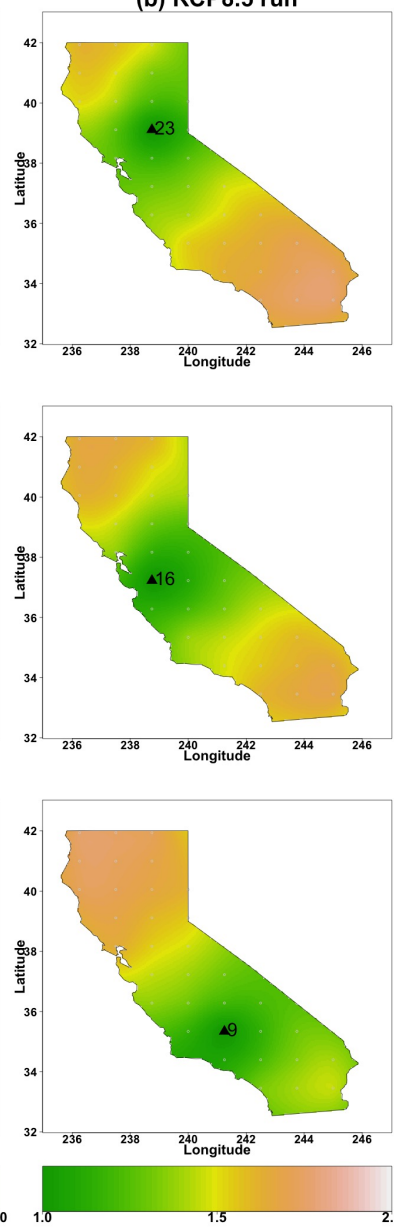

(c) Difference: historical-RCP8.5
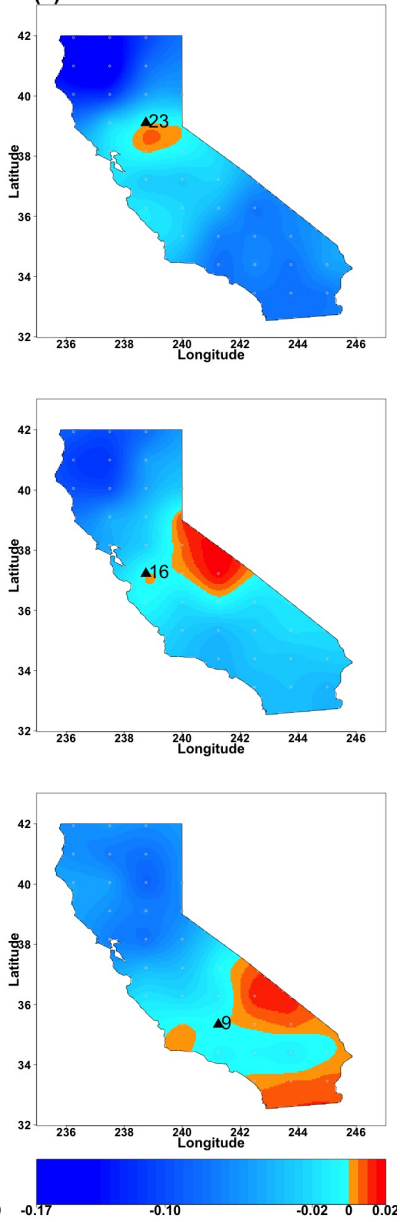

Figure 6. Ensemble means of pairwise extremal coefficients of annual maximum AR precipitation from a focal grid point (black triangle; grid locations 23, 16, and 9 from top to bottom) to other locations in California for CMIP5 multi-models. Changes are shown over two 25-year time periods - (a) 1981-2005 and (b) 2076-2100 - as well as (c) the difference.

model ensembles. Pairwise extremal coefficients (which range from 1 to 2) are estimated between the black triangle point and the other grid points and plotted with a color gradient for the grid points 23, 16, and 9 in Fig. 5a. The coefficient is 1 (green) in the case of perfect dependence and increases to 2 (tan-white) when two locations are completely independent, i.e., not spatially correlated. Extremal coefficients from the focal grid point to areas beyond the grid points are estimated using kriging, a method of statistical interpolation, and colors corresponding to the extremal coefficient estimates are plotted all over the California region. Differences in the ensemble average between the historical run (1981-2005) and the future run (2076-2100) are illustrated in Fig. 6c. Blue and red colors represent decreases (negative values in range of -0.17 to 0 ) and increases (positive values in range of 0 0.02) in spatial dependence under global warming, respectively. The decreases (blue) in spatial dependence represent AR precipitation extremes that are spatially less correlated under the high future emissions scenario, while the increases (red) represent more spatially correlated AR extreme precipitation than in the historical run.

The range of spatial dependence (green area) is concentrated within a smaller localized area in California for the future under the highest emissions scenario than for the current climate. In particular, the range of strong dependence from a focal grid point in northern California to other points becomes narrower than the range of dependence from the focal grid points in central or southern California under RCP8.5. Though we arbitrarily selected three focal locations as representative of the three regions of California, the decreasing pattern of dependence range is true for other focal locations as well. The blue colors in the difference plots of Fig. 6 show a less spatially correlated pattern of annual maximum AR precipitation between a focal grid point and other grid points in California in a warming scenario. Small areas of grid points near focal grid points show increases (i.e., red colors in the difference plots of Fig. 6) in spatial dependence. Therefore, we postulate that future extreme precipitation dur- 
(a) Historical: lower bound
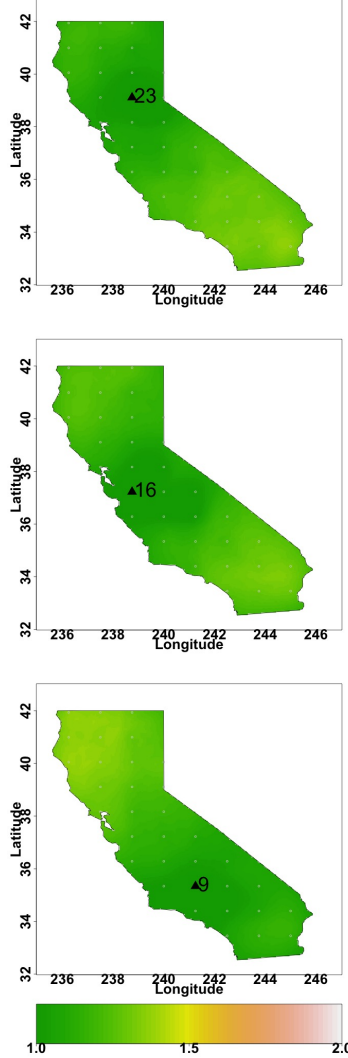

(b) Historical: upper bound
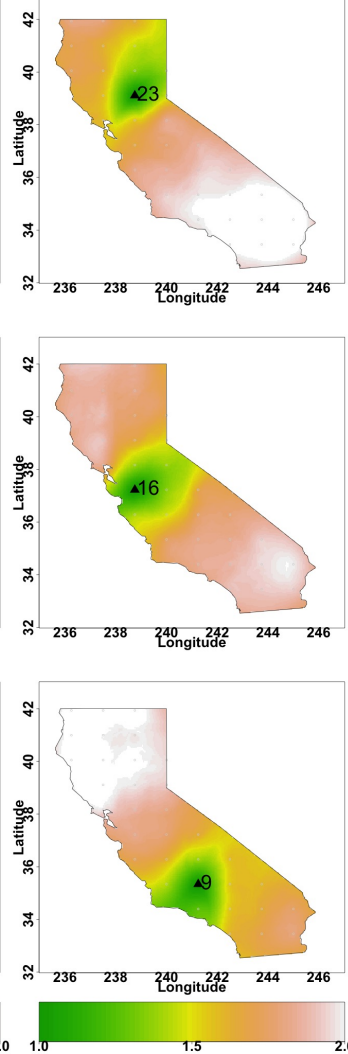

(c)
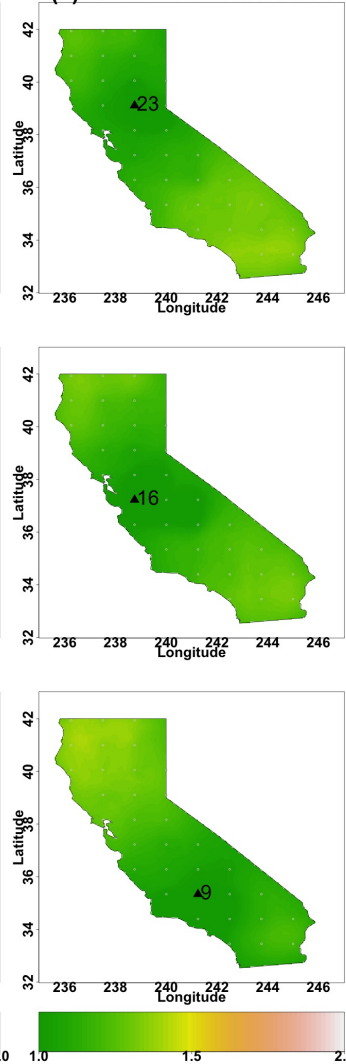

(d) RCP8.5: upper bound
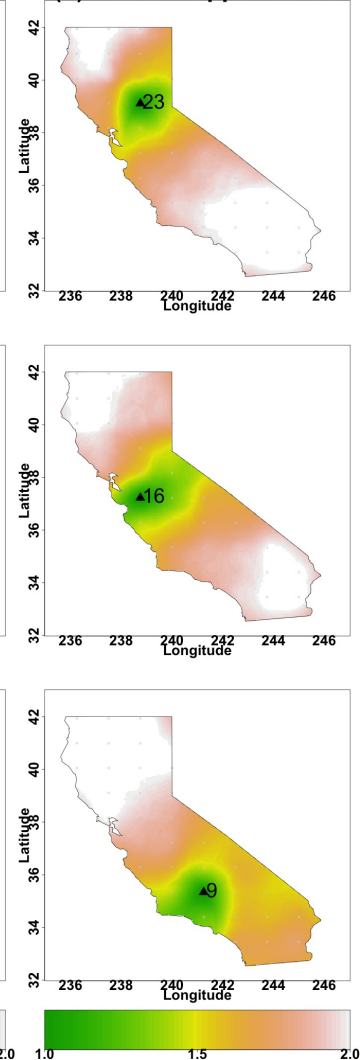

Figure 7. Lower ((a) for historical run and (c) RCP8.5 run) and upper bounds ((b) for historical run and (d) RCP8.5 run) of $95 \%$ confidence intervals for the ensemble means of pairwise extremal coefficients in Fig. 6 for CMIP5 multi-model ensembles. Black triangle points represent the grid locations 23,16 , and 9 from top to bottom.

ing ARs will be less spatially correlated than in the current climate. However, the negative changes in multi-model ensemble means of spatial dependence are not statistically significant at the $5 \%$ significance level and the changes of dependence pattern remain uncertain.

Figure 7 shows the lower and upper bounds of the $95 \%$ confidence interval for ensemble means of pairwise extremal coefficients from both the historical and RCP8.5 runs. The confidence interval to account for the uncertainty in point estimation of extremal coefficients is obtained from 500 bootstrap samplings with all possible multi-model ensemble members and time replication. The confidence interval is narrower for areas near a focal location representing small uncertainty, whereas the confidence interval is wider in distant areas from the focal grid point. Compared to the upper bounds in Fig. 7, trends in decreases of spatial dependence are more obvious at a focal grid point near northern California than near southern California.

To summarize pairwise spatial dependence visually over the entire grid, we transform the extremal coefficients to the values between 0 (complete independence) and 1 (complete dependence) by a simple calculation. We propose an arbi- trary value, 1.3 , as a threshold of strong dependence, and count the number of values with strong dependence $(<1.3)$ at each grid point. The strong dependence $(<1.3)$ shows bigger changes which are easier to capture than mild dependence, about 1.5 , so the arbitrary threshold of 1.3 is chosen as the value of strong dependence. The count takes the values between 0 and the number of grid points, not including the focal grid point in the model. Then, we normalize the count by the total number of points to attain the same scale for all models and color the grid box based on the fraction, as shown in Fig. 8. For example, the value 0 represents any location that does not show strong dependence (relative to the threshold) with the grid point in question. If the grid point is strongly dependent for all other 33 locations (showing extremal coefficients smaller than 1.3), the fraction is 1 .

Figure 8 shows color changes as more orangish, yellowish, and yellowish green over the region, representing more independent patterns among maximum AR precipitation within a warmer climate. Specifically, the decreases (more orangish and yellowish color in the warmer future) in spatial dependence are obvious in northern California from multimodel ensemble means. There are also some squares near 
Table 3. Summary: Change in atmospheric river properties from multi-model ensemble outputs under late 21 st century RCP8.5 forcing compared to the recent past (historical). There are increases in AR days, AR frequency, and heavy rainfall associated with ARs, while spatial dependence between the annual maximum AR precipitation decreases in the future under a warming scenario. The $95 \%$ confidence interval (CI) is calculated for the difference in means (RCP8.5 run - historical run) of each AR property.

\begin{tabular}{|c|c|c|c|c|}
\hline & Historical (1981-2005) & RCP8.5 (2076-2100) & Changes $(\%)$ & $95 \% \mathrm{CI}$ \\
\hline AR days (days $\mathrm{yr}^{-1}$ ) & 14.5 & 19.3 & +33 & $(2.57,7.07)$ \\
\hline AR frequency (events $\mathrm{yr}^{-1}$ ) & 10.6 & 14.7 & +39 & $(2.54,5.68)$ \\
\hline AR precipitation extremes $\left(\mathrm{mm} \mathrm{day}^{-1}\right)$ & 45.1 & 51.3 & +14 & $(0.94,11.73)$ \\
\hline Range of dependence $\left(\theta_{1}\right)$ & 4.6 & 3.7 & -20 & $(-1.73,0.04)$ \\
\hline
\end{tabular}

(a) Historical run

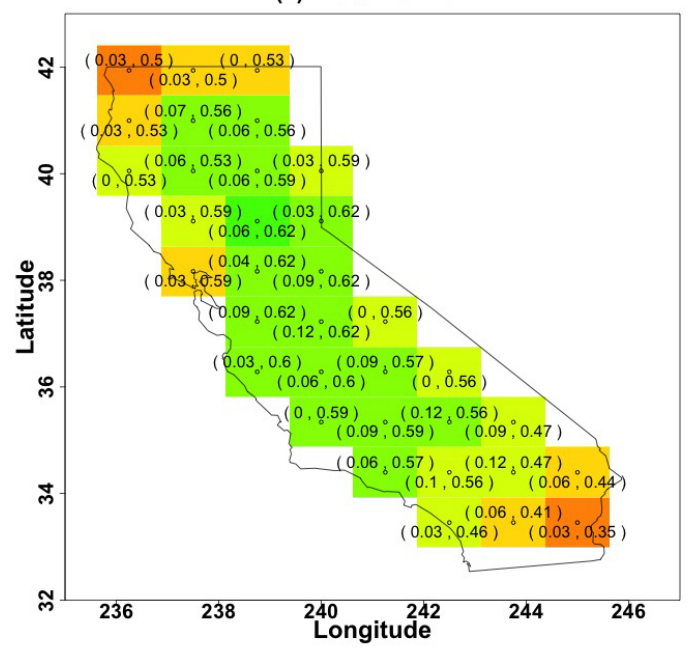

(b) RCP8.5 run

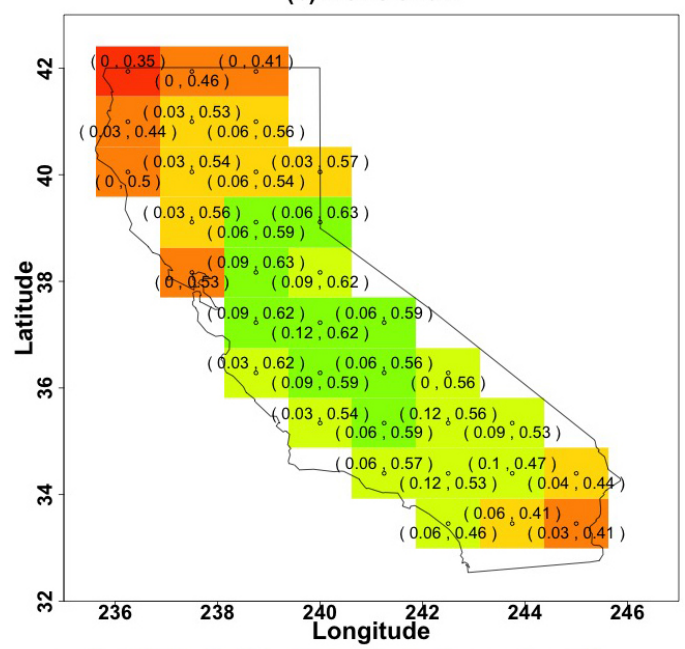

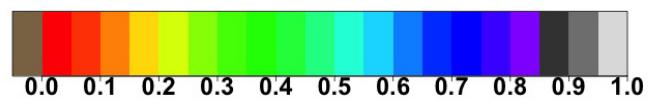

Figure 8. A summary of the changes in pairwise spatial dependence from (a) 1981-2005 to (b) 2076-2100 for multi-model ensemble simulations. Discrete colors at each location represent the number of locations with strong dependence (i.e., extremal coefficient $<1.3$ ). The values in parenthesis at each color box represent lower and upper bounds of the $95 \%$ confidence interval for the number of locations.

the coast and southern squares showing decreases (changes from greenish to yellowish green color) under the RCP8.5 scenario. Based on a two-sample permutation test, this analysis provides statistically significant evidence of the changes in ensemble mean of the number of locations showing strong dependence $(p$ value $=0.005)$. The values in parenthesis in each color box in Fig. 8 represent the lower and upper bounds of the $95 \%$ confidence interval for the count of locations showing strong dependence. The bounds of the interval represent the uncertainty in estimating the number of locations related to strong dependence. Here, interpreting the upper bound is more valuable than interpreting the lower bound, as it provides a suggestion of possible limits of the values. Upper bounds in the RCP8.5 scenario are also smaller than those in the historical run in the northern California area.

\section{Conclusions}

We have studied the influence of ARs on the spatial coherence of extreme precipitation under a changing climate. We have detected AR events using the TECA framework and investigated changes in properties of ARs such as total AR days, frequency, intensity of precipitation extremes associated with ARs, and spatial dependence patterns of extreme rainfall in multi-model ensemble means from CMIP5 simulations. A brief summary is provided in Table 3 . We find there are significant increases in AR days, AR frequency, and occurrence of heavy rainfall under future RCP8.5 scenarios. We show that the spatial dependence between extreme precipitations during ARs decreases in future, warmer climates. Although future ARs produce more severe rainfall, different AR events might bring extreme rainfall intensity that is less spatially correlated under the warming scenario. However, the changes in spatial dependence are not significant, which 
implies large uncertainty regarding the decreases of spatial dependence between AR extreme precipitations from model outputs.

Our current analysis remains preliminary due to the limited data availability from the CMIP5 models. More GCM simulations need to be considered to characterize changes in AR properties and the behavior of tail dependence across models. Furthermore, simulated extreme precipitation amounts from the relatively coarse horizontal resolutions of the CMIP5 models are substantially lower than in the real world (Wehner et al., 2014). Lavers et al. (2013) discussed projected changes in ARs, suggesting that these changes are thermodynamic responses to a warming climate from anthropogenic radiative forcing. Further studies on the physical and dynamical processes of ARs are necessary to better understand the changes in these storms in warmer climate ARs as well as in their impacts on California.

Acknowledgements. This research was supported by the Director, Office of Science, Office of Advanced Scientific Computing Research, of the US Department of Energy under contract no. DE-AC02-05CH11231. This work was funded under the CASCADE Science Focus Area funded by DOE/BER as part of their Regional and Global Climate Modeling Program. This research used resources of the National Energy Research Scientific Computing Center.

Edited by: X. Zhang

Reviewed by: two anonymous referees

\section{References}

Bernard, E., Naveau, P., Vrac, M., and Mestre, O.: Clustering of Maxima: Spatial Dependencies among Heavy Rainfall in France, J. Climate, 26, 7929-7937, 2013.

Byna, S., Prabhat, Wehner, M. F., and Wu, K. J.: Detecting Atmospheric Rivers in Large Climate Datasets, in: Proceedings of the 2nd International Workshop on Petascal Data Analytics: Challenges and Opportunities, PDAC '11, 7-14, ACM, New York, NY, USA, doi:10.1145/2110205.2110208, 2011.

Coelho, C. A. S., Ferro, C. A. T., Stephenson, D. B., and Steinskog, D. J.: Methods for Exploring Spatial and Temporal Variability of Extreme Events in Climate Data, J. Climate, 21, 2072-2092, 2008.

Coles, S. G.: An Introduction to Statistical Modeling of Extreme Values, Springer Verlag, New York, USA, 45-91 , 2001.

Cooley, D., Naveau, P., and Poncet, P.: Variograms for spatial maxstable random fields, Lecture Notes in Statistics, 187, 373-390, 2006.

de Haan, L.: A spectral representation for max-stable processes, Ann. Probab., 12, 1194-1204, 1984.

Dettinger, M. D.: Fifty-two years of "pineapple-express" storms across the West Coast of North America, Tech. rep., US Geological Survey, Scripps Institution of Oceanography for the California Energy Commission, PIER Project Rep, cEC-500-2005-004, 20 pp., 2004.
Dettinger, M. D.: Climate Change, Atmospheric Rivers, and Floods in California - A Multimodel Analysis of Storm Frequency and Magnitude Change, J. Am. Water Resour. Assoc., 47, 514-523, 2011.

Furrer, E. M., Katz, R. W., Walter, M. D., and Furrer, R.: Statistical modeling of hot spells and heat waves, Clim. Res., 43, 191-205, 2010.

Grotjahn, R. and Faure, G.: Composite Predictor Maps of Extraordinary Weather Events in the Sacramento California Region, Weather Forecast., 23, 313-335, 2008.

Kabluchko, Z., Schlather, M., and de Haan, L.: Stationary maxstable fields associated to negative definite functions, Ann. Probab., 37, 2042-2065, 2009.

Kharin, V. V., Zwiers, F. W., Zhang, X., and Hegerl, G. C.: Changes in Temperature and Precipitation Extremes in the IPCC Ensemble of Global Coupled Model Simulations, J. Climate, 20, 14191444, doi:10.1175/JCLI4066.1, 2007.

Kharin, V. V., Zwiers, F. W., Zhang, X., and Wehner, M. F.: Changes in temperature and precipitation extremes in the CMIP5 ensemble, Clim. Change, 119, 345-357, 2013.

Langford, S., Stevenson, S., and Noone, D.: Analysis of LowFrequency Precipitation Variability in the CMIP5 Historical Simulations for Southwestern North America, J. Climate, 27, 27352756, 2014.

Lavers, D. A., Villarini, G., Allan, R. P., Villarini, G., Wood, E. F., and Wade, A. J.: The detection of atmospheric rivers in atmospheric reanalyses and their links to British winter floods and the large-scale climatic circulation, J. Geophys. Res., 117, D20106, doi:10.1029/2012JD018027, 2012.

Lavers, D. A., Allan, R. P., Villarini, G., Lloyd-Hughes, B., Brayshaw, D. J., and Wade, A. J.: Future changes in atmospheric rivers and their implications for winter flooding in Britain, Environ. Res. Lett., 8, 034010, 2013.

Leung, L. R. and Qian, Y.: Atmospheric rivers induced heavy precipitation and flooding in the western U.S. simulated by WRF regional climate model, Geophys. Res. Lett., 36, L03820, doi:10.1029/2008GL036445, 2009.

Lundquist, J. D., Minder, J. R., Neiman, P. J., and Sukovich, E.: Relationships between Barrier Jet Heights, Orographic Precipitation Gradients, and Streamflow in the Northern Sierra Nevada, J. Hydrometeorol., 11, 1141-1156, 2010.

Naveau, P., Guillou, A., Cooley, D., and Diebolt, J.: Modeling pairwise dependence of maxima in space, Biometrika, 96, 1-17, 2009.

Neiman, P. J., Ralph, F. M., Wick, G. A., Lundquist, J. D., and Dettinger, M. D.: Meteorological characteristics and overland precipitation impacts of atmospheric rivers affecting the West Coast of North America based on eight years of SSM/I satellite observations, J. Hydrometeorol., 9, 22-47, 2008.

Payne, A. E. and Magnusdottir, G.: Dynamics of Landfalling Atmospheric Rivers over the North Pacific in 30 Years of MERRA Reanalysis, J. Climate, 27, 7133-7150, 2014.

Prabhat, Rubel, O., Byna, S., Wu, K., Li, F., Wehner, M., and Bethel, W.: TECA: A Parallel Toolkit for Extreme Climate Analysis, Procedia Computer Science, 9, 866-876, doi:10.1016/j.procs.2012.04.093, 2012.

Ralph, F. M., Neiman, P. J., and Wick, G. A.: Satellite and caljet aircraft observations of atmospheric rivers over the eastern north 
pacific ocean during the winter of 1997/98, Mon. Weather Rev., 132, 1721-1745, 2004.

Ralph, F. M., Neiman, P. J., and Rotunno, R.: Dropsonde observations in low-level jets over the Northeastern Pacific Ocean from CALJET-1998 and CALJET-2001: Mean vertical-profile and atmospheric-river characteristics, Mon. Weather Rev., 133, 889-910, 2005.

Ralph, F. M., Neiman, P. J., Wick, G. A., Gutman, S. I., Dettinger, M. D., Cayan, D. R., and White, A. B.: Flooding on California's Russian River: Role of atmospheric rivers, Geophys. Res. Lett., 33, L13801, doi:10.1029/2006GL026689, 2006.

Rutz, J. J., Steenburgh, W. J., and Ralph, F. M.: Climatological Characteristics of Atmospheric Rivers and Their Inland Penetration over the Western United States, Mon. Weather Rev., 142, 905-921, doi:10.1175/MWR-D-13-00168.1, 2014.

Schlather, M.: Models for stationary max-stable random fields, Extremes, 5, 33-44, 2002.

Schlather, M. and Tawn, J. A.: A dependence measure for multivariate and spatial extreme values: Properties and Inference, Biometrika, 90, 139-156, 2003.

Sillmann, J., Kharin, V. V., Zwiers, F. W., Zhang, X., and Bronaugh, D.: Climate extremes indices in the CMIP5 multimodel ensemble - Part 1. Model evaluation in the present climate, J. Geophys. Res. Atmos., 118, 1716-1733, doi:10.1002/jgrd.50203, $2013 \mathrm{a}$.

Sillmann, J., Kharin, V. V., Zwiers, F. W., Zhang, X., and Bronaugh, D.: Climate extremes indices in the CMIP5 multimodel ensemble - Part 2. Future climate projections, J. Geophys. Res. Atmos., 118, 2473-2493, doi:10.1002/jgrd.50188, 2013b.
Warner, M. D., Mass, C. F., and Salathé Jr., E. P.: Wintertime Extreme Precipitation Events along the Pacific Northwest Coast: Climatology and Synoptic Evolution, Mon. Weather Rev., 140, 2021-2043, 2012.

Wehner, M. F.: Very extreme seasonal precipitation in the NARCCAP ensemble: Model performance and projections, Clim. Dynam., 40, 59-80, 2013.

Wehner, M. F., Reed, K. A., Li, F., Prabhat, Bacmeister, J., Chen, C.-T., Paciorek, C., Gleckler, P. J., Sperber, K. R., Collins, W. D., Gettelman, A., and Jablonowski, C.: The effect of horizontal resolution on simulation quality in the Community Atmospheric Model, CAM5.1, J. Adv. Model. Earth Sys., 6, 980-997, 2014.

Weller, G. B., Cooley, D. S., and Sain, S. R.: An investigation of the pineapple express phenomenon via bivariate extreme value theory, Environmetrics, 23, 420-439, 2012.

Wick, G. A., Neiman, P. J., and Ralph, F. M.: Description and Validation of an Automated Objective Technique for Identification and Characterization of the Integrated Water Vapor Signature of Atmospheric Rivers, IEEE Trans. Geosci. Remote Sens., 51, 2166-2176, 2013.

Wu, K., Otoo, E., and Suzuki, K.: Optimizing Two-pass Connectedcomponent Labeling Algorithms, Pattern Anal. Appl., 12, 117135, doi:10.1007/s10044-008-0109-y, 2009. 